\title{
Formation of ring-shaped microtubule assemblies through active self-organization on dynein
}

\author{
Masaki Ito $^{1}$, Arif Md. Rashedul Kabir ${ }^{2}$, Daisuke Inoue ${ }^{1}$, Takayuki Torisawa ${ }^{3}$, Yoko Toyoshima ${ }^{3}$, \\ Kazuki Sada ${ }^{1,4}$ and Akira Kakugo ${ }^{1,4,5}$
}

Microtubule (MT)-kinesin, a biomolecular motor system, is a promising candidate for construction of artificial biomachines for a variety of nanotechnology applications. An active self-organization (AcSO) method involving a specific streptavidin (St)-biotin (Bt) interaction has been developed to assemble MTs into a highly ordered structure by exploiting their motility on a kinesincoated surface. Dynein is another biomolecular motor that moves along the MTs in the opposite direction from kinesin. Dynein has not yet been used to demonstrate the AcSO of MTs. In this study, we report the first successful demonstration of the AcSO of MTs on a dynein-coated surface to produce ring-shaped MT assemblies similar to those of kinesin. We found that ring-shaped MT assemblies obtained on dynein showed equal clockwise and counterclockwise rotational motion. This work will enrich the building blocks for designing future oriented motor protein-based artificial devices.

Polymer Journal (2014) 46, 220-225; doi:10.1038/pj.2013.89; published online 18 December 2013

Keywords: active self-organization; dynein; microtubule; ring-shaped assemblies

\section{INTRODUCTION}

In living cells, biomolecular motor systems such as actin-myosin and microtubule (MT)-kinesin are important for activities such as motility, ${ }^{1}$ cell division ${ }^{2}$ and cellular transport. ${ }^{3}$ Biomolecular motor systems are well known for their high efficiency in converting chemical energy into mechanical work with high specific power (power per unit mass ${ }^{4}$ ), which is the reason that bimolecular motors have been proposed as the building blocks for nanobiodevices.

The highly processive biomolecular motor kinesin, which moves MTs toward their plus end, has widely been employed in nanoscale molecular shuttles, ${ }^{5,6}$ surface imaging tools, ${ }^{7}$ force measurements ${ }^{8,9}$ and lab-on-a-chip sensor devices. To integrate the MT-kinesin system into more ordered structures that might provide emergent functions as realized in natural systems, we have recently developed the method of active self-organization (AcSO) using a specific streptavidin (St)-biotin (Bt) interaction during the sliding motion of MTs on a kinesin-fixed surface in the presence of adenosine-tri-phosphate (ATP). ${ }^{10-12}$ This ATP-driven energydissipative self-organization process reaches a steady state that is far from equilibrium, differentiating it from conventional self-assembly processes that always tend to stay at an equilibrium state. This energydissipative AcSO method introduced a wide variety of motile MT assemblies of different shapes, such as bundles, networks and rings, in response to different experimental conditions, such as changes in MT and kinesin concentrations and St-Bt labeling ratio. ${ }^{13,14}$ Ring-shaped
MT assemblies were found to show rotational motion without changing the position of the center of mass, and because of the prospective utilization of continuous networks in future nanotechnological applications, these ring-shaped MT assemblies have attracted much attention. ${ }^{10-12,15-18}$

Dynein is another biomolecular motor that moves along MTs using the chemical energy of ATP and critically differs from kinesin with respect to the direction of movement along MTs. ${ }^{19-21}$ Introduction of dynein to $\mathrm{AcSO}$, which has not yet been addressed, will widen the future application of biomolecular motor systems. In this paper, we have demonstrated the first AcSO of MTs on a dynein-coated surface. We found that, as in the case of kinesin, AcSO using dynein also successfully produced ring-shaped MT assemblies under certain experimental conditions. Our work thus provides a means for enriching the collection of organized MT assemblies, which in turn will enhance the development of biomolecular motor-based artificial biomachines.

\section{EXPERIMENTAL PROCEDURE}

\section{Tubulin purification}

Tubulin was purified from porcine brain using a high-concentration 1,4piperazinediethanesulfonic acid (PIPES; Sigma, Tokyo, Japan) buffer (1 M PIPES, 20 mм EGTA, $10 \mathrm{~mm} \mathrm{MgCl}_{2}$; $\mathrm{pH}$ adjusted to 6.8); high-molarity PIPES (HMPB) and BRB80 buffers ( $80 \mathrm{~mm}$ piperazine-N,N'-bis(2-ethanesulfonic acid), $2 \mathrm{~mm} \mathrm{MgCl}_{2}$ and $1 \mathrm{~mm}$ EDTA at $\mathrm{pH}$ 6.9) were prepared using PIPES, and the $\mathrm{pH}$ was adjusted using $\mathrm{KOH}$.

${ }^{1}$ Graduate School of Chemical Sciences and Engineering, Hokkaido University, Sapporo, Japan; ${ }^{2}$ Faculty of Science, Hokkaido University, Sapporo, Japan; ${ }^{3}$ Department of Life Sciences, Graduate School of Arts and Sciences, The University of Tokyo at Komaba, Tokyo, Japan; ${ }^{4}$ Graduate School of Science, Hokkaido University, Sapporo, Japan and 5PRESTO, Japan Science and Technology Agency, Saitama, Japan

Correspondence: Dr A Kakugo, Department of Chemistry, Faculty of Science, Hokkaido University, Sapporo 060-0810, Japan. E-mail: kakugo@sci.hokudai.ac.jp

Received 9 April 2013; revised 2 September 2013; accepted 4 October 2013; published online 18 December 2013 


\section{Dynein purification}

Cytoplasmic dynein was purified from porcine brain according to a published procedure. $^{22}$ The anion exchange column (UnoQ-1; Bio-Rad Laboratories, Hercules, CA, USA)-purified cytoplasmic dynein peaks were pooled in HPLC buffer ( $35 \mathrm{~mm}$ tris(hydroxymethyl)aminomethane), $5 \mathrm{~mm} \mathrm{MgSO}$, 1 mM EGTA, $0.5 \mathrm{~mm}$ EDTA, 1 mм DTT, $10 \mathrm{~mm}$ ATP, $\mathrm{pH}$ 7.2) and supplemented with $24 \%$ sucrose. Dynein was flash-frozen and stored in liquid nitrogen.

\section{Bt labeling of tubulin and stoichiometric estimation}

Bt-labeled tubulin was prepared using biotin-XX-SE (Invitrogen/Life Technologies Japan, Tokyo, Japan) as previously described. ${ }^{23}$ The labeling stoichiometry was $\sim 0.6$ per tubulin heterodimer, which was estimated by spectrometric titration using 2-(4'-hydroxyphenylazo)-benzoic acid (HABA) dye (Wako, Tokyo, Japan). ${ }^{24}$

\section{MT preparation}

Bt and rhodamine-labeled MTs were obtained by polymerizing a mixture of Bt-tubulin and rhodamine-tubulin (Bt/Tub: 0.6; final tubulin concentration: $70 \mu \mathrm{M})$ in a polymerization buffer ( $80 \mathrm{~mm}$ PIPES, $1 \mathrm{~mm}$ EGTA, $5 \mathrm{~mm} \mathrm{MgCl}_{2}$, $1 \mathrm{~mm}$ GTP, $\sim 1 \%$ DMSO; pH adjusted to 6.8 using $\mathrm{KOH})$ at $37^{\circ} \mathrm{C}$. After the prescribed incubation time ( $30 \mathrm{~min}, 24 \mathrm{~h}$ ), the MTs were diluted to make the tubulin concentration $100 \mathrm{~nm}$ in a buffer $(80 \mathrm{~mm}$ PIPES-2K, $1 \mathrm{~mm}$ EGTA, $1 \mathrm{~mm}$ $\mathrm{MgCl}_{2}, 0.13 \mathrm{M}$ DMSO, $10 \mu \mathrm{M}$ taxol; $\mathrm{pH}$ adjusted to 6.8 using $\mathrm{HCl}$ ).

\section{Formation of ring-shaped MT assemblies through AcSO}

To perform the in situ observation of MT-ring formation, a motility assay was carried out inside a humidified inert gas chamber system. ${ }^{25}$ The inert gas chamber system consists of three main parts: a nitrogen gas cylinder, a humidity supporter and the chamber. The chamber has two segments: a base plate made of stainless steel and a cover plate made of poly(methyl methacrylate). The base plate contains a horizontally placed inlet and outlet, which facilitates the passage of nitrogen gas through the chamber and allow the flow rate of nitrogen gas to be controlled. To prevent the MTs from drying and to prevent damage by reactive oxygen species, we used the inert gas chamber system during motility assays.

Flow cells were prepared by placing a small cover glass $\left(5 \times 5 \mathrm{~mm}^{2}\right.$; Matsunami, Tokyo, Japan) on a large slide glass $\left(40 \times 50 \mathrm{~mm}^{2}\right)$ equipped with a pair of spacers to form a chamber of $\sim 4 \times 5 \times 0.1 \mathrm{~mm}^{3}(W \times L \times H)$ in dimension. Plasma treatment was applied on the glass surface to obtain a hydrophilic surface.

The flow cell was filled with $5 \mu$ l of casein solution ( $80 \mathrm{~mm}$ PIPES- $2 \mathrm{~K}, 1 \mathrm{~mm}$ EGTA, $1 \mathrm{mM} \mathrm{MgCl}_{2}, \sim 0.2 \mathrm{mg} \mathrm{ml}^{-1}$ casein; $\mathrm{pH}$ adjusted to 6.8 using $\mathrm{HCl}$ ) to prevent the adhesion of dynein active domains directly on the glass surface. After incubating for $3 \mathrm{~min}$ with casein solution to mask the glass surface, $5 \mu \mathrm{l}$ of $60 \mathrm{~nm}$ cytoplasmic-dynein solution ( $\sim 80 \mathrm{~mm}$ PIPES, $\sim 40 \mathrm{~mm} \mathrm{NaCl}, 1 \mathrm{~mm}$ EGTA, $1 \mathrm{mM} \mathrm{MgCl}_{2}, 0.5 \mathrm{mg} \mathrm{ml}^{-1}$ casein, $1 \mathrm{~mm}$ DTT, $10 \mu \mathrm{M}$ taxol, $\sim 0.13 \mathrm{M}$ DMSO; $\mathrm{pH}$ 6.8) was introduced and incubated for $3 \mathrm{~min}$. The flow cell was washed with $5 \mu \mathrm{l}$ of motility buffer (80 mM PIPES, 1 mM EGTA, $2 \mathrm{~mm} \mathrm{MgCl}_{2}$, $0.5 \mathrm{mg} \mathrm{ml}^{-1}$ casein, $1 \mathrm{~mm}$ DTT, $10 \mu \mathrm{M}$ paclitaxel, and $\sim 0.13 \mathrm{M}$ DMSO; $\mathrm{pH}$ 6.8). A diluted solution $(5 \mu \mathrm{l})$ of MTs ( $100 \mathrm{~nm}$ in motility buffer) was then introduced and incubated for $5 \mathrm{~min}$, followed by additional washing with $5 \mu \mathrm{l}$ of motility buffer. The partial coverage of Bt on the MT surface with St (Wako) was performed by incubating the MTs with St solution (10 nM in motility buffer) for $3 \mathrm{~min}$ after fixing the MTs on dynein. Finally, the motility assay was initiated by applying $24 \mu \mathrm{l}$ of ATP solution (motility buffer supplemented with $5 \mathrm{~mm}$ ATP). All of the above procedures were conducted at room temperature.

\section{Microscopic image analysis of motility assays}

Rhodamine-labeled MT assemblies in motility assays were illuminated with a $100-\mathrm{W}$ mercury lamp and visualized by an epi-fluorescence microscope with a Plan Apo $60 \times / 1.40$ objective (Nikon, Tokyo, Japan). Images were captured using a cooled-CCD camera (iXon; ANDOR Technology, Tokyo, Japan) connected to a PC.

\section{Image analysis for motility assays}

Movies and images of the motility assays captured by fluorescence microscopy were analyzed to determine the velocity of the MTs using the image analysis software (ImageJ, offered by the National Institutes of Health (NIH) and available at: http://rsb.info.nih.gov/ij/). Although the inner surface of the cover glass was observed through the glass, the captured images correspond to those observed from the reverse side of the flow cell. The clockwise (CW)/counterclockwise (CCW) motion was discussed based on the captured images. In fluorescence microscopic images, one pixel corresponded to $0.11 \mu \mathrm{m}$ as a resolution. Velocity measurements were conducted using the software by manually tracking inhomogeneities, such as defects where MTs were partly winded down or differences in the fluorescence intensities of the MT rings. To measure the velocity of the MT rings, the tracks of the MTs in the six sequential images in $5 \mathrm{~s}$ intervals were manually detected using the software.

\section{RESULTS AND DISCUSSION}

Before performing the AcSO of MTs using dynein, we investigated the mobility of MTs, which is necessary for successful self-organization on a dynein-coated cover glass surface. In our study, the hydrophilicity of the glass surface appeared to be an important factor in ensuring the dynein-based motility of MTs. Strong hydrophobic interaction between the dynein and the cover glass surface seems to hinder the activity of dynein, perhaps due to the mode of adsorption of dynein that prevented MTs from approaching the active site of the motor protein and consequently resulting in failure of MT motility. The hydrophilicity of the glass surface was increased dramatically by applying a plasma treatment, as shown in Figure 1. To optimize the effect of the plasma treatment on the motility of MTs, we measured the velocity of MTs on cover glass surfaces that were plasma treated for different lengths of time (Figure 2). The velocity of MTs increased with time of plasma treatment and reached a plateau after $30 \mathrm{~s}$ of treatment. On the basis of this result, we consistently applied the plasma treatment to the cover glass surface for $60 \mathrm{~s}$ in this study.

AcSO of MTs was then performed on the plasma-treated cover glass surface coated with dynein by employing a specific St-Bt interaction through the in vitro motility assay. The AcSO of MTs performed on a dynein-coated cover glass surface is schematically shown in Figure 3. MTs were polymerized from tubulin monomers modified with Bt by incubating for $30 \mathrm{~min}$ at $37^{\circ} \mathrm{C}$, and the labeling stoichiometry was kept at 0.6 per tubulin heterodimer. After a flow cell was prepared, dynein was immobilized on the plasma-treated cover glass surface, a

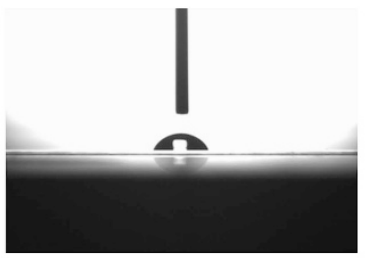

c

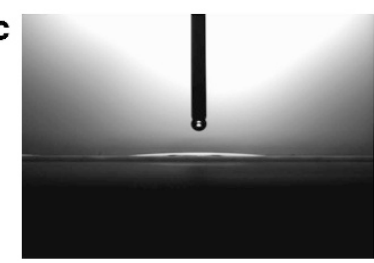

b

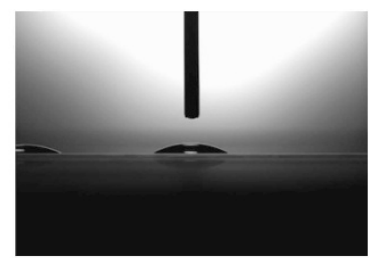

d

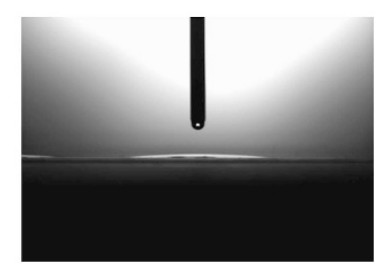

Figure 1 Hydrophilicity of different glass surfaces: (a) cover glass without any treatment, (b) $1 \mathrm{~s}$ plasma-treated cover glass, (c) $10 \mathrm{~s}$ plasma-treated cover glass and (d) 20 s plasma-treated cover glass. 


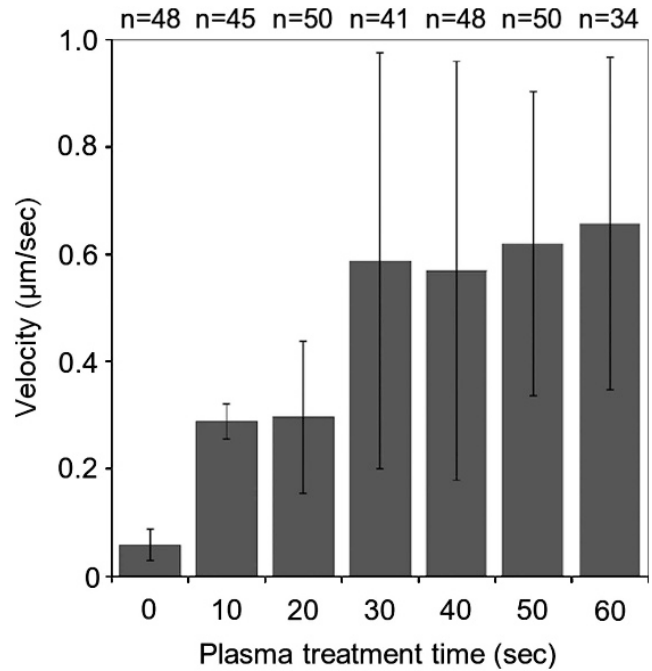

Figure 2 Effect of plasma treatment time of cover glass on the velocity of microtubules (MTs) on dynein-coated glass surfaces, where $n$ is the number of MTs considered for analyses.
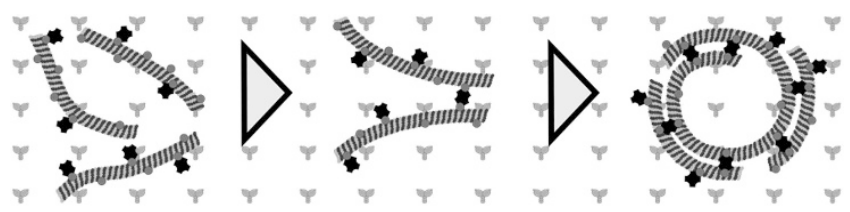

: Dynein /IIIf: Microtubule ๑: Biotin Streptavidin

Figure 3 Schematic representation of the active self-organization (AcSO) of microtubules (MTs) on a dynein-coated surface. A full color version of this figure is available at Polymer Journal online.

and MTs were then applied to the flow cell. On the basis of our previous report, ${ }^{26}$ the MT concentration and St/Bt ratio were held at $100 \mathrm{~nm}$ and $1 / 10$, respectively, to allow the formation of ring-shaped MT assemblies on the dynein-fixed surface. By adding ATP to the flow cell, we initiated the AcSO of the MTs. To permit the motility of the MTs for a longer amount of time, the flow cell was placed in an inert chamber after the addition of ATP and was incubated in the inert chamber by flowing humid nitrogen. ${ }^{27,28}$ The AcSO of the MTs was monitored under a fluorescence microscope, and sample observation was performed $1 \mathrm{~h}$ after ATP addition. Figure 4 shows a representative fluorescence image of the MT assemblies after $1 \mathrm{~h}$ of ATP addition. As shown in the figure, most of the MTs were found to form ring-shaped structures. The number density of the ring-shaped MT assemblies was 6.87 per $100 \mu \mathrm{m}^{2}$, which was almost unchanged even after performing the AcSO for $>1 \mathrm{~h}$. This result indicates that MT ring formation reached a stable state by $1 \mathrm{~h}$ of AcSO. The percentage of ring-shaped MT assemblies showed that rotational motion was almost $100 \%$ (movie is available in Supporting information/movie 1). The mean velocity of the ring-shaped $\mathrm{MT}$ assemblies was found to be $0.04 \pm 0.002 \mu \mathrm{m} \mathrm{s}^{-1}$. A histogram of the inner diameter of the ringshaped MT assemblies is shown in Figure 5, with a mean diameter of $4.86 \pm 3.29 \mu \mathrm{m}$. Next, we investigated the direction of the rotational motion of those ring-shaped MT assemblies. We previously reported ${ }^{26}$ that ring-shaped MT assemblies formed through AcSO on a kinesincoated cover glass surface showed preferential rotational motion

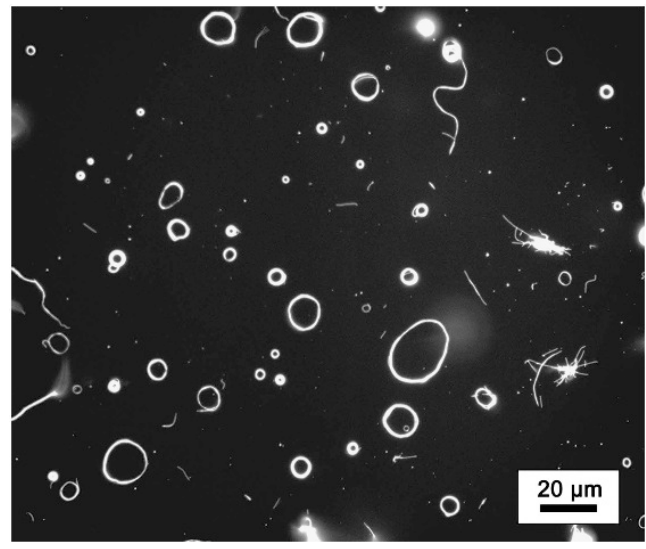

Figure 4 Fluorescence microscopy image of the ring-shaped microtubule (MT) assemblies formed through the active self-organization (AcSO) on a dynein-coated surface. A full color version of this figure is available at Polymer Journal online.

(CW:CCW $=21: 79)$. In that report, MT filaments were prepared by incubating tubulin monomers for $30 \mathrm{~min}$ at $37^{\circ} \mathrm{C}$, and the same conditions were used in the present work. However, as shown in Figure 6, ring-shaped MT assemblies obtained on the dynein-coated surface showed no preference in the direction of rotational motion. The percentages of ring-shaped MT assemblies rotating in CW and CCW directions were found to be almost equal. We also previously reported that ring-shaped MT assemblies obtained through AcSO on kinesin showed preferential rotation in the CCW direction (CW:CCW $=73: 26$ ) when the AcSO was performed using MT polymerized for $24 \mathrm{~h}$ at $37^{\circ} \mathrm{C}$. In the case of dynein, we observed that the ring-shaped MT assemblies obtained through the AcSO of MTs prepared by polymerizing for $24 \mathrm{~h}$ at $37^{\circ} \mathrm{C}$ did not show any preferential rotation, CW:CCW $=40: 60$ (Figure 6). Thus, results obtained in the present study using dynein are in contrast to the results observed in the previous report using kinesin. ${ }^{26}$ Moreover, we also observed no difference in the velocity of the ring-shaped MT assemblies moving in CW and CCW directions on the dynein-coated surface, and this result was consistent with that obtained in the case of kinesin. ${ }^{26}$ Next, the rotational direction and inner diameter of the ring-shaped MT assemblies formed on a dynein-coated surface were compared with the aforementioned results of the previous study (Figure 7). As shown in this figure, any obvious change in the distribution of the inner diameters of the rings moving CCW and CW cannot be found. Previously, it was shown that on a kinesin-coated surface, the direction of the rotational motion of ring-shaped MT assemblies could be tuned from CCW to CW by using structurecontrolled MTs. ${ }^{26,29}$ However, in the present study, ring-shaped MT assemblies formed on a dynein-coated surface did not show any directional preference despite the use of structure-controlled MTs. The different behaviors of ring-shaped MT assemblies observed in this and the previous study could be explained by the different interaction mechanisms of kinesin and dynein with MTs. Kinesin is known to be a motor protein that moves along the MT in a highly coordinated manner because of its high processivity. In contrast, the less processive dynein has been known to show a highly variable stepping pattern. ${ }^{30,31} \mathrm{MT}$ is a cylindrical protein composed of protofilaments (PFs) of alpha- and beta-tubulin subunits where longitudinal interactions between the subunits are basically hydrophobic, and lateral interaction of inter-PFs is electrostatic due to the interactions 


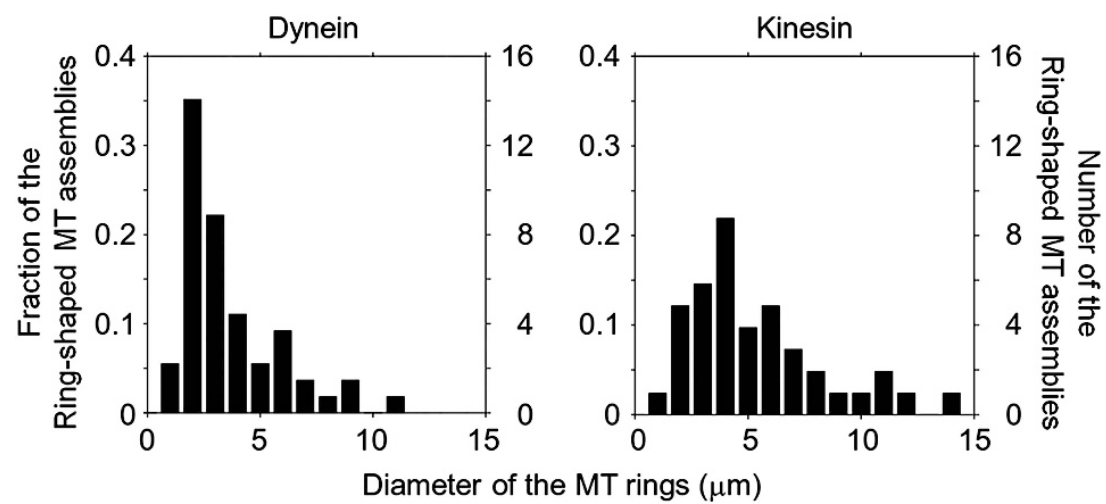

Figure 5 Size distribution of ring-shaped microtubules (MTs) formed through the active self-organization (AcSO) on dynein- and kinesin-coated surfaces. MTs polymerized for $30 \mathrm{~min}$ at $37^{\circ} \mathrm{C}$ were used in these experiments.

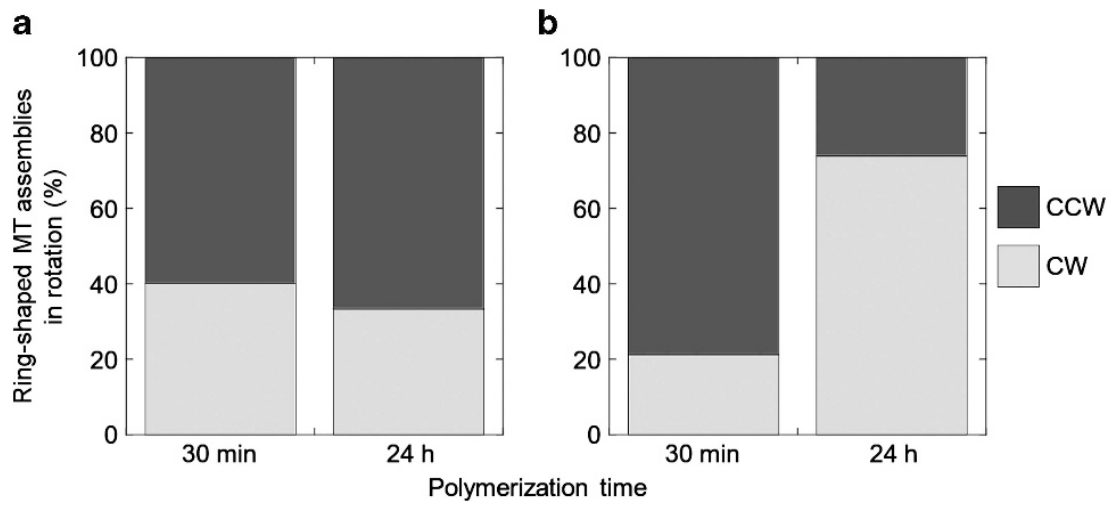

Figure 6 Effect of polymerization time of microtubule (MTs) on the preferential rotation of ring-shaped MTs formed by active self-organization (AcSO) on (a) dynein- and (b) kinesin-coated surfaces.
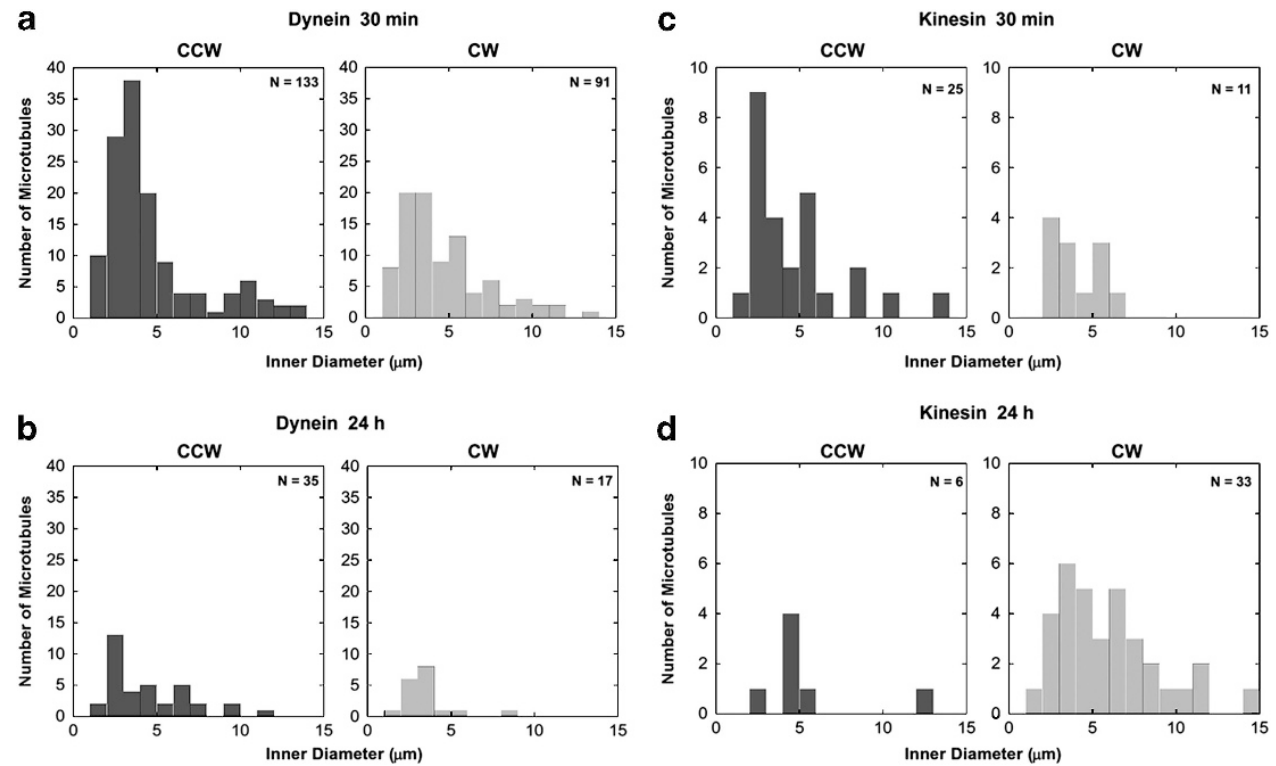

Figure 7 Preferential rotation and size distributions of ring-shaped microtubule (MT) assemblies formed through active self-organization (AcSO) on dyneinand kinesin-coated surfaces. The times for MT polymerization were $30 \mathrm{~min}$ and $24 \mathrm{~h} . \mathrm{N}$ is the number of MT rings considered for analyses the motor protein and polymerization time are described as follows (a) dynein, $30 \mathrm{~min}$, (b) dynein, $24 \mathrm{~h}$, (c) kinesin, $30 \mathrm{~min}$, (d) kinesin, $24 \mathrm{~h}$. 
a

Microtubule with left-handed supertwist
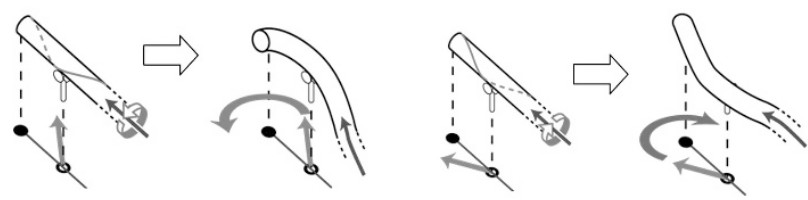

Figure 8 Schematic illustration showing the movement of a single microtubule (MT) on a kinesin-coated surface and the possibility of inducing a biased rotation resulting from the helical structure of protofilaments (PFs). The bending directions are opposite for MTs with (a) left- and (b) righthanded supertwists. A full color version of this figure is available at Polymer Journal online.

Table 1 Summarized data on the velocity, inner diameter and direction of rotation of ring-shaped MT assemblies formed through AcSO on dynein- and kinesin-coated surfaces

\begin{tabular}{lccccc}
\hline & \multicolumn{2}{c}{ Dynein } & & \multicolumn{2}{c}{ Kinesin } \\
\cline { 2 - 3 } \cline { 5 - 6 } Incubation time & $30 \mathrm{~min}$ & $24 \mathrm{~h}$ & & $30 \mathrm{~min}$ & $24 \mathrm{~h}$ \\
\hline Velocity $\left(\mu \mathrm{ms}^{-1}\right)$ & $0.03 \pm 0.02$ & $0.07 \pm 0.05$ & $0.20 \pm 0.04$ & $0.24 \pm 0.04$ \\
Inner diameter $(\mu \mathrm{m})$ & $4.87 \pm 3.22$ & $4.14 \pm 0.30$ & & $4.56 \pm 2.52$ & $5.82 \pm 2.97$ \\
Rotation $(\mathrm{CCW} / \mathrm{CW}(\%))$ & $40 / 60$ & $33 / 67$ & & $21 / 79$ & $74 / 26$
\end{tabular}

Abbreviations: AcSO, active self-organization; CCW, counterclockwise; CW, clockwise; MT, microtubule.

of amino acids. The longitudinal interaction is affected by temperature and $\mathrm{pH}$ change, whereas the lateral one is affected by a change in the dielectric constant of the medium..$^{32}$ The incubation time for polymerizing MTs is also important for allowing the MTs to reach their most energetically favorable configuration. ${ }^{33}$ It has been reported that the number of PFs settled within a lattice of MTs is responsible for the left- or right-handed supertwist of MTs, minimizing the restoring force in the lattice. ${ }^{34}$ In our previous study, we reported that with an increase in incubation time during MTs polymerization, the number of PFs within the MTs lattice shifted toward larger values. This increase in the number of PFs is correlated with the shift of left-handed supertwist in the MT lattice toward a right-handed supertwist. In this study, as shown in Figure 8, an MT with a left- or right-handed supertwist shows axial rotation (green arrow) when moving over processive motor protein. ${ }^{29}$ This axial rotation is assumed to be superimposed on the linear motion (blue arrow), generating a torque (violet arrow) on the MT that leads to bending of the front segment of MT. The handedness of the torque might finally determine the direction of rotation of ring-shaped MT assemblies formed. In the case of a less processive motor protein, the supertwist structure of MTs might have a less pronounced effect in determining the handedness of torque on moving MTs. Consequently, the bending direction of the MT tends to be more stochastically averaged, and the ring-shaped MT assemblies showed unbiased rotational motion.

We also found that the inner diameter distribution of the ringshaped MT assemblies formed on dynein (mean inner diameter $=4.13 \pm 2.22 \mu \mathrm{m}$ ) was almost the same as that of assemblies formed on kinesin (mean inner diameter $=5.82 \pm 2.97 \mu \mathrm{m}$ ). It is unclear why smaller rings were formed on dynein. Further investigations will be performed in future work. We have summarized data on the velocity distribution, inner diameter and direction of rotational motion of ring-shaped MT assemblies formed on a dynein-coated surface using MTs prepared by polymerizing tubulin for $30 \mathrm{~min}$ and $24 \mathrm{~h}$ at $37^{\circ} \mathrm{C}$. We also compared our data with that obtained from the AcSO using kinesin (Table 1).

In conclusion, for the first time, we have successfully demonstrated the AcSO of MTs on a dynein-coated cover glass surface. The AcSO produced ring-shaped MT structures that showed nonpreferential rotational motion. Our work brings variation to the collection and design of organized MT assemblies that should foster the development of biomolecular motor-based artificial biomachines.

\section{ACKNOWLEDGEMENTS}

This research was financially supported by the Ministry of Education, Science, Sports, and Culture of Japan (Grant-in-Aid of Specially Promoted Scientific Research).

1 Hirokawa, N. Kinesin and dynein superfamily proteins and the mechanism of organelle transport. Science 279, 519-526 (1998).

2 Umeda, M. \& Emoto, K. Membrane phospholipid dynamics during cytokinesis: regulation of actin filament assembly by redistribution of membrane surface phospholipid. Chem. Phys. Lipids 101, 81-91 (1999).

3 Bachand, M., Trent, A., Bunker, B. \& Bachand, G. Physical factors affecting kinesin-based transport of synthetic nanoparticle cargo. J. Nanosci. Nanotechnol. 5, 718-722 (2005)

4 Visscher, K., Schnitzer, M. \& Block, S. Single kinesin molecules studied with a molecular force clamp. Nature 400, 184-189 (1999).

5 Turner, D., Chang, C., Fang, K., Cuomo, P. \& Murphy, D. Kinesin movement on glutaraldehyde-fixed microtubules. Anal. Biochem. 242, 20-25 (1996).

6 Ramachandran, S., Ernst, K.-H., Bachand, G., Vogel, V. \& Hess, H. Selective loading of kinesin-powered molecular shuttles with protein cargo and its application to biosensing. Small. 2, 330-334 (2006)

7 Hess, H., Clemmens, J., Howard, J. \& Vogel, V. Surface Imaging by Self-Propelled Nanoscale Probes. Nano. Lett. 2, 113-116 (2002).

8 Hess, H., Howard, J. \& Vogel, V. A Piconewton Forcemeter assembled from microtubules and kinesins. Nano Lett. 2, 1113-1115 (2002).

9 van den Heuvel, M. \& Dekker, C. Motor proteins at work for nanotechnology. Science 317, 333-336 (2007).

10 Hess, H., Clemmens, J., Brunner, C., Doot, R., Luna, S., Ernst, K.-H. \& Vogel, V. Molecular self-assembly of "nanowires"and "nanospools" using active transport. Nano Lett. 5, 629-633 (2005).

11 Kawamura, R., Kakugo, A., Osada, Y. \& Gong, J. Selective formation of a linear-shaped bundle of microtubules. Langmuir 1, 533-537 (2010).

12 Kakugo, A., Shikinaka, K., Matsumoto, K., Gong, J. \& Osada, Y. Growth of large polymer-actin complexes. Bioconjug. Chem. 14, 1185-1190 (2003).

13 Tamura, Y., Kawamura, R., Shikinaka, K., Kakugo, A., Osada, Y., Gong, J. \& Mayama, H. M. Dynamic self-organization and polymorphism of microtubule assembly through active interactions with kinesin. Soft Matter 7, 5654-5659 (2011).

14 Kawamura, R., Kakugo, A., Osada, Y. \& Gong, J. Microtubule bundle formation driven by ATP: the effect of concentrations of kinesin, streptavidin and microtubules. Nanotechnology 21, 145603-145614 (2010).

15 Hess, H. Self-assembly driven by molecular motors. Soft Matter 2, 669-677 (2006).

16 Liu, H., Spoerke, E. D., Bachand, M., Koch, J. S., Bunker, C. B. \& Bachand, D. G. Biomolecular motor-powered self-assembly of dissipative nanocomposite rings. Adv. Mater. 20, 4476-4481 (2008)

17 Luria, I., Crenshaw, J., Downs, M., Agarwal, A., Seshadri, B. S., Gonzales, J., Idan, O., Kamcev, J., Katira, P., Pandey, S., Nitta, T., Phillpot, R. S. \& Hess, H. Microtubule nanospool formation by active self-assembly is not initiated by thermal activation. Soft Matter 7, 3108-3115 (2011).

18 Idan, O., Lam, A., Kamcev, J., Gonzales, J., Agarwal, A. \& Hess, H. Nanoscale transport enables active self-assembly of millimeter-scale wires. Nano Lett. 12, 240-245 (2012)

19 Kural, C., Kim, H., Syed, S., Goshima, G., Gelfand, V. I. \& Selvin, P. R. Kinesin and dynein move a peroxisome in vivo: a tug-of-war or coordinated movement? Science 308, 1469-1472 (2005).

20 Derr, N. D., Goodman, B. S., Jungmann, R., Leschziner, A. E., Shih, W. M. \& ReckPeterson, S. L. Tug-of-war in motor protein ensembles revealed with a programmable DNA origami scaffold. Science 338, 662-665 (2012).

21 Blehm, B. H., Schroer, T. A., Trybus, K. M., Chemla, Y. R. \& Selvin, P. R. In vivo optical trapping indicates kinesin's stall force is reduced by dynein during intracellular transport. Proc. Natl Acad. Sci. USA 110, 3381-3386 (2013). 
22 Toba, S. \& Toyoshima, Y. Dissociation of double-headed cytoplasmic dynein into singleheaded species and its motile properties. Cell. Motil. Cytoskeleton. 58, 281-289 (2004).

23 Hyman, A., Drechsel, D., Kellogg, D., Salser, S., Sawin, K., Steffen, P., Wordeman, L. \& Mitchison, T. Preparation of modified tubulins. Methods Enzymol. 196, 478-485 (1991).

24 Green, N. Spectrophotometric determination of avidin and biotin. Methods Enzymol. 18, 418-424 (1970).

25 Kabir, A. M. R., Inoue, D., Kakugo, A., Kamei, A. \& Gong, J. P. Prolongation of the active lifetime of a biomolecular motor for in vitro motility assay by using an inert atmosphere. Langmuir 27, 13659-13668 (2011).

26 Kakugo, A., Kabir, A. M. R., Hosoda, N., Shikinaka, K. \& Gong, J. P. Controlled clockwise-counterclockwise motion of the ring-shaped microtubules assembly. Biomacromolecules 12, 3394-3399 (2011).

27 Kabir, A. M. R., Inoue, D., Kakugo, A., Kamei, A. \& Gong, J. P. Prolongation of the active lifetime of a biomolecular motor for in vitro motility assay by using an inert atmosphere. Langmuir 27, 13659-13668 (2011).
28 Kabir, A. M. R., Inoue, D., Kakugo, A., Sada, K. \& Gong, J. P. Active self-organization of microtubules in an inert chamber system. Polym. J. 44, 1-5 (2012).

29 Kawamura, R., Kakugo, A., Shikinaka, K., Osada, Y. \& Gong, J. P. Ring-shaped assembly of microtubules shows preferential counterclockwise motion. Biomacromolecules 9, 2277-2282 (2008).

30 Quu, W., Derr, N., Goodman, B., Villa, E., Wu, D., Shih, W. \& Reck-Peterson, S. Dynein achieves processive motion using both stochastic and coordinated stepping. Nat. Struct. Mol. Biol. 19, 193-200 (2012).

31 DeWitt, M., Chang, A., Combs, P. \& Yildiz, A. Cytoplasmic dynein moves through uncoordinated stepping of the AAA + ring domains. Science 335, 221-225 (2012).

32 Nogales, E. Tubulin rings: which way do they curve? Curr. Opin. Struct. Biol. 13, 256-261 (2003).

33 Hunyadi, V., Chretien, D., Flyvbjerg, H. \& Janosi, I. M. Why is the microtubule lattice helical? Biol. Cell 99, 117-128 (2007).

34 Chrétien, D. \& Fuller, S. Microtubules switch occasionally into unfavorable configurations during elongation. J. Mol. Biol. 298, 663-676 (2000). 